\title{
Understanding health behaviour changes in response to outbreaks: findings from a longitudinal study of a large epidemic of mosquito-borne disease
}

\section{Authors and affiliations:}

Jocelyn Raude ${ }^{1,2, *}$

Kathleen MCColl1,2

Claude Flamand ${ }^{3}$

Themis Apostolidis 4

1) EHESP Rennes, Université Sorbonne Paris Cité, France;

2) Unité des Virus Emergents (UVE: Aix-Marseille Univ - IRD 190 - Inserm 1207 - IHU Méditerranée Infection), Marseille, France;

3) Institut Pasteur de Guyane, Unité d’Epidémiologie, Cayenne, France;

4) Aix Marseille Univ, LPS, Aix-en-Provence, France.

\section{*Corresponding author:}

Jocelyn Raude, PhD, Department of Social and Behavioral Sciences, EHESP French School of Public Health, 15 Avenue du Professeur Leon-Bernard, CS 74312, 35043 Rennes Cedex, France.

Tel.: +330299022615

Email: Jocelyn.Raude@ehesp.fr

\section{Funding support:}

Financial support for this study was provided in part by grants from the French National Institute of Health and Medical Research (under the agreement number IMMI2014001). The funding agreement ensured the authors' independence in designing the study, interpreting the data, writing, and publishing the report. 


\begin{abstract}
Rationale. Although greater attention has been recently given to the ecological determinants of health behaviours, we still do not know much about the behavioural changes induced by the spread of infectious diseases.

Objective. In this study, we took advantage of a large epidemic of chikungunya, an emerging mosquito-borne disease, in French Guiana to examine the dynamic interaction between risk-related perceptions and behaviours that occurs in response to a disease outbreak. In particular, we tested empirically the assumption that both risk perceptions and health behaviours were elastic with respect to prevalence of chikungunya.
\end{abstract}

Methods. A representative sample of French Guianan $(N=434)$ was interviewed in January 2015 just after the peak of the epidemic, and again 2 months later. Participants were asked about their perceptions of the threat, as well as their engagement in a range of protective behaviours promoted by the regional health authorities to control the spread of the disease.

Results. The surveys showed that (1) the frequency of some health behaviours - those related to visible control methods - significantly increased with the subjective and objective prevalence of the disease, (2) perceived risk of infection for oneself tended to decrease considerably over time, and (3) the risk reappraisal hypothesis failed to account for this paradoxical trend in the people's response to the risk of contracting the disease.

Conclusion. These findings suggest that people may fail to adjust their risk perceptions, and to a lesser extent their health protective behaviours, to the course of an epidemic. Notably, the prevalence elasticity of preventive action found in previous studies of behavioural response to infectious diseases differed substantially according to the type of intervention (personal versus environmental methods). This paradoxical trend may be attributed to risk habituation effects, which seem to vary significantly according to the social visibility of the preventive actions. 
Keywords: Behaviour change, epidemics, risk perception, risk habituation, elasticity-prevalence, mosquito-borne diseases

\section{Introduction}

During the last decade, an important theoretical shift within the epidemiological and biomathematical literature has been observed, resulting in the increasing recognition by modellers of the key role of human behaviours in the spread of infectious diseases. Recently, several leading authors in the field have -advocated that understanding the dynamics of infectious disease transmission requires a more fine-grained approach that takes into account how epidemics influence human behaviours, and how epidemics are in turn influenced by behavioural changes (Cherif et al., 2016; Ferguson, 2007; Funk et al., 2015). Indeed, from a psychological perspective, it is difficult to conceive that risk perceptions and behaviours would remain constant in the face of epidemiological events. As stressed by Rogers (1997, p. 752): “Postulating a static risk perception would involve describing mechanisms by which people continue to hold-fast to views of risk in the face of new information, particularly when risk events with potentially life threatening consequences are considered." In response to such criticism, many epidemiological modellers have recently attempted to take into account better the reciprocal influences between environments and behaviours in their models (Funk et al., 2010; Weston et al., 2018). Notably, as an infectious disease spreads, it is assumed that individuals tend to adopt health protective behaviours that impact upon the rate at which the disease spreads. In spite of these advances, a recent systematic review conducted by Verelst and his colleagues shows that most studies present in silico models, which do not involve the use of empirical data in models of behavioural change and in parameters of peopleenvironment interactions. For these authors (Verelst et al., 2016, p. 1), the fact that "most models 
are purely theoretical and lack representative data" raises serious concern about their accuracy and relevance.

Despite growing evidence of their importance in infectious disease transmission, little is known about whether and how human behaviours are shaped by epidemics. To date, only a limited number of empirical investigations have addressed this critical issue (Xu and Peng, 2015), most of which concluded that changes in health protective behaviours generally seem to reflect by and large changes in disease prevalence. In behavioural economics, this relationship between disease prevalence and health protective behaviours is called "elasticity-prevalence" of prevention-related decisions (Geoffard and Philipson, 1996; Philipson, 2000). In the nineties, several authors therefore attempted to calculate coefficients of elasticity-prevalence for a given preventive behaviour in response to a given disease such as influenza or malaria (Pattanayak et al., 2007; Picone et al., 2013; Seban et al., 2013). However, there is still little empirical evidence to support the elasticityprevalence of preventive behaviours. As noted by Pattanayak and his colleagues (Pattanayak et al., 2006), elasticity-prevalence effects reported in the literature have mainly been found through cross-sectional studies based on aggregated data at the regional or country level. Further, alternative hypotheses drawn from the health psychological literature - particularly risk denial and risk habituation - have been largely ignored in studies devoted to individual and community responses to epidemics, probably because there is still a considerable lack of longitudinal data in health behaviour research (Sutton, 2004).

In this study, we take advantage of a large outbreak of chikungunya virus (CHIKV) infection, a re-emerging mosquito-borne disease that struck French Guiana in 2014-15, in order to examine the dynamic nature of risk perceptions and health protective behaviours in response to an epidemic. More specifically, longitudinal data were collected using a within-individuals prospective design with two waves of measurement to test the following assumption empirically: 1) both risk judgments and health protective behaviours are elastic to prevalence of CHIKV infection, and 2) if 
this were not the case, whether an apparent lack of elasticity in the perceived risk could be attributed to the risk reappraisal effect. It is hoped that the results obtained from the current study will help both psychologists and epidemiologists understand better the complex interactions among epidemiological, cognitive and behavioural factors that occur within an epidemic setting.

\section{Theoretical Background}

Throughout the last decades, the majority of studies devoted to individual variations in behavioural responses to a health threat has focused on personal/cognitive determinants rather than social/ecological determinants of actions or habits that relate to health promotion and disease prevention (Golden and Earp, 2012; Sallis et al., 2015). Of these personal factors, risk perceptions have been consistently found to play an important role in the adoption of health behaviours that reduce the risk of developing a disease such as a cancer or a coronary disease (Brewer et al., 2004; Ferrer and Klein, 2015; Sheeran et al., 2014). This theoretical orientation reflects a predominance of health behaviour research focused on non-communicable diseases, for which the main epidemiological parameters such as incidence, prevalence, and mortality are likely to remain relatively stable over time. This may have contributed to overlooking the role played by the natural environment in the adoption of health protective behaviours. As far as infectious diseases are concerned, these features are rarely static, so the behavioural response to a threat is likely to change as an epidemic progresses (Weston et al., 2018). This raises the following question: what are people's possible responses to a significant change in the epidemiological context? In a seminal article on the dynamics of risk perception, Loewenstein and Mather (Loewenstein and Mather, 1990) explored a variety of possible relationships between epidemiological and cognitive factors. In particular, based on longitudinal studies, they described typical patterns about how perceived risk may change during an epidemic (see figure 1). 
1. Accuracy of risk perceptions: this pattern occurs when perceived risk of infection is found to reflect fairly accurately observable changes within the epidemiological context (e.g., the mortality due to a specific disease). As noted by Fergusson (2007, p. 733): "Individuals are most likely to change their contact patterns when mortality or the perception of risk is high, and resume normal life as the perceived risk declines". This process is somewhat equivalent to the concept of behavioural plasticity in ecology, and the prevalence-elasticity concept in behavioural economics, mentioned above.

2. Risk habituation: this pattern refers to a phenomenon of adaptation whereby people tend to underestimate progressively or neglect risks as a health threat becomes increasingly familiar. Whilst habituation effects are well-established in the psychology and physiology literature, there is however little empirical evidence that risk perception is also subject to such an effect (Lima, 2004).

3. Public panic: this pattern is described by Loewenstein and Mather as (1990, p. 171) "a sudden surges of concern that are unrelated to, or vastly out of proportion to changes in the underlying problem." In other words, panic occurs when the perceived risk related to a given disease temporarily rises to a level which is overtly disproportionate to, or far exceeds, the objective epidemiological parameters.

Despite the undisputable interest of this work, one must nonetheless proceed with caution in generalising these results to the actions taken by individuals or communities to reduce their risk, as behavioural changes do not necessarily track the evolution of risk perception. In a series of theoretical and empirical studies, Neil Weinstein and Noel Brewer have shown that there is a complex interaction between the perceived risk of becoming infected and the adoption of health protective behaviours over time (Brewer et al., 2007a, 2004; Weinstein and Nicolich, 1993). Firstly, they found that an initial perception of a high risk of future infection motivated people to engage in health protective behaviours (the motivational effect). Second, they showed that people were likely 
to lower their risk perception after taking protective action considered as effective to prevent or control disease transmission (the risk reappraisal effect). Third, the authors found that people who engaged in some protective behaviours believed that, as a result of their actions, their personal risk of infection was lower than those who did not (the accuracy of comparative risk perception effect). Overall, the combination of these different effects explains why researchers are likely to form misleading or incorrect conclusions about the relation between cognitive and behavioural factors from cross-sectional studies. For instance, a shift in risk perception as the epidemic of chikungunya progresses could be interpreted erroneously as a risk denial process when in reality, it may be caused by the adoption of protective behaviours that objectively reduce the risk of subsequent infection. This highlights the need for longitudinal research into the relationship between risk perception and protective behaviours over time through the course of the epidemic.

\section{Environmental and Epidemiological Setting}

French Guiana is a small French overseas region located in the northeast coast of South America. It is covered in tropical rainforest and its population of approximately 280,000 is located mainly on the coast, with half the population residing in the capital, Cayenne. Whilst basic infrastructure is relatively poor and the cost of living and crime rate are high, as it is a French department, inhabitants benefit not only from a good health care service, but also from subsidies, trade, goods and services from the French mainland. Nonetheless, it is important to note that the unemployment rate exceeds $20 \%$ and $40 \%$ of the population lives below the poverty threshold. Considered as a monsoon climate, the hot and humid weather, along with heavy rainfall, provide an ideal environment for the breeding of mosquitoes responsible for the transmission of CHIKV, malaria, dengue or dengue fever. Apart from these mosquito-borne diseases, public health warnings issued for French Guiana regularly include HIV, hepatitis A and B, typhoid, and to a lesser extent, 
rabies. However, it should be noted that only a small number of positive cases of these infectious diseases were diagnosed and confirmed in laboratory during the chikungunya epidemic that hit French Guiana in 2014/15.

Chikungunya is an arboviral disease caused by CHIKV, an alphavirus transmitted to humans mainly through the bite of an infected Aedes mosquito (Burt et al., 2012; Lo Presti et al., 2014; Pialoux et al., 2007; Robinson, 1955). It is an acute infection with an asymptomatic incubation period lasting on average between two to three days. Clinical onset is abrupt with symptoms generally resolving within seven to ten days. The illness is usually self-limiting and spontaneously resolves with time. Nevertheless, complications, and indeed chronic complications such as rheumatic disorders are common. In particular, CHIKV is frequently associated with persistent and severe polyarthralgia 6-12 months after infection. In Reunion Island, $97 \%$ of patients complained of lasting symptoms longer than 6 months after infection (Simon et al., 2011). In Jamaica, 70\% of the CHIKV-infected adults reported arthralgia 12 months after the disease outbreak (O'Sullivan et al., 2018). Currently, there is neither pharmaceutical treatment nor available vaccine to prevent the risk of infection with CHIKV.

Since 2004, the virus has spread at an unprecedented rate, leading to epidemics in Africa, Asia, Europe, as well islands in the Indian Ocean (Burt et al., 2012; Rougeron et al., 2015). Prior to December 2013, despite numerous imported cases and the presence of the main vectors $A$. Albopictus and A. Aegypti, CHIKV transmission had not been documented in the Americas(Bajak, 2014; Weaver, 2014). In December 2013, however, autochthonous cases were detected on the Island of Saint Martin where the virus spread rapidly, leading to a local transmission of CHIKV in the Caribbean and the Americas, including Florida and Puerto Rico (Bajak, 2014; Cassadou et al., 2014). After the identification of some clusters of autochthonous cases in summer 2014, a large outbreak occurred in French Guiana during the winter of 2014-15 (see figure 2) (Flamand et al., 
2017). According to a recent sero-epidemiological study, about one quarter of the adult population had been infected by CHIKV (Fritzell et al., 2019). As Aedes mosquitoes have become more and more resistant to conventional chemical interventions, public health authorities have developed an Integrated Vector Management (IVM) strategy which, through education and communication campaigns, promotes the adoption of protective behaviours among populations exposed to the competent vectors (WHO, 2004). Indeed, behavioural changes in the populations exposed to vectorborne diseases are increasingly viewed as a critical factor in the development of effective programs and policies aiming to prevent or reduce their transmission (Elder and Lloyd, 2006).

\section{Material and Methods}

To investigate the complex interactions among epidemiological, cognitive and behavioural factors that occur in an epidemic setting, a two-wave longitudinal design was employed, with a first wave of data collection performed in January 2015 (baseline interview), shortly after the peak of the epidemic was reached), and a second wave of data collection performed 2 months later (followup interview), before the substantial decrease in weekly CHIKV incidence (see figure 2). The hypotheses tested in this study are derived from the possible effects of change in disease prevalence on risk perceptions and health protective behaviours that are summarized in the figure 1. Given the large proportion of the populations affected by chikungunya outbreaks, it was expected that most participants in our surveys had experienced the disease, either directly through a personal history of CHIKV infection or indirectly through the observation and discussion of cases of these illnesses which occurred within their social circles, as well as the intensive media coverage of the epidemic by regional newspapers, radio, and television.

\section{Participants and Procedure}


The data were collected in French Guiana in January 2015 by a professional survey company (IPSOS) by means of computer-assisted telephone interviews (CATI) of inhabitants aged 18 and over. A proportional random digit dialling method was used to recruit survey participants across the country. Furthermore, a stratified selection procedure based on the administrative area population (communes and counties) was used to ensure the representativeness of the sample. Gender, age and occupational status of respondents were also controlled by using quotas so that the sample approximated the latest regional Census data. The interview acceptance rate - which took into account people who were contacted but refused to be interviewed - was 59\%, yielding to an initial sample size of 768 for a total population of about 280,000. Participants were interviewed again for the follow-up (Wave 2) in April 2015. The follow-up completion rate was 57\%, yielding a final sample of 434 individuals. As shown in table 1, no significant difference was observed in the sociodemographic characteristics of the participants between the baseline and follow-up survey. Moreover, as there was no significant difference in the psychological and behavioural variables of interest between the respondents and non-respondents, we used complete case analysis (CCA) rather than multiple imputation (MI) techniques and ignored the incomplete observations from the participants missing in the follow-up sample (Sidi and Harel, 2018).

This study was approved by the National Committee of personal data protection (Commission National Informatique \& Liberté) which is the authority in charge of ethical considerations in social and behavioural research in France. As informed consent cannot be expressed in writing within telephone survey, participants' oral consent was sought in accordance with the principles of research involving human subjects established by the Declaration of Helsinski.

\section{Measures}




\section{Sociodemographic and illness-related variables}

The questionnaire included a wide range of items aimed at collecting socioeconomic and demographic information such as age, gender, education, marital status, occupational status, size of household and housing conditions. In addition, participants were asked whether (1) over the last months they had personally suffered from a range of symptoms (high fever, severe joint pain, swelling, skin rash, headache) that could indicate an infection by CHIKV (response options: 'Yes', 'No', or 'Not sure') and whether (2) another member of their household had recently suffered from these symptoms (response options: 'Yes', 'No', or 'Not sure'), and if 'yes', how many (open-ended question). These last items were used to estimate the symptomatic prevalence of chikungunya in Waves 1 and 2.

\section{Perceptions of risk related to chikungunya}

To capture changes in risk-related beliefs in epidemic settings, we used several conceptions and measures, ranging from the deliberative to the affective approach of risk perception, based upon leading psychological model of health behaviour (such as the Health Belief Model, the Protection Motivation Theory, or the Tripartite Model of Risk Perception) and currently employed in the relevant literature (Dillard et al., 2012; Ferrer et al., 2013; Weinstein et al., 2007). First, the perceived prevalence of CHIKV was assessed with one item adapted from Viscusi (Raude et al., 2018): “Among 100 people living in French Guiana, how many do you think have been infected with chikungunya since the beginning of the epidemic? Please give a value between 0 and 100". Second, the participants were asked about their personal risk of infection with a series of 5 items (e.g., "What is the risk that you will get the chikungunya before the end of the epidemic", "How worried are you about getting chikungunya?", "How vulnerable do you feel to chikungunya”?). With the exception of perceived prevalence, the format and phrasing of these items were adapted from Weinstein (Weinstein, 2000), and rated on an 11-point Likert scale ranging from 0 to 10 in which 
the meaning of the end-point values was explicitly indicated. The responses were then summed across items to generate scores on a personal risk perception scale (Cronbach's alpha=0.69 and 0.72 in Wave 1 and 2, respectively).

\section{Health protective behaviours}

Human behaviours aimed at reducing exposure to mosquitoes and mosquito-borne diseases can be divided into two categories: biochemical and physical interventions. In order to capture the change in health protective behaviours, participants were questioned in the baseline and follow-up surveys about the frequency of 9 health protective behaviours promoted by regional public health authorities to reduce the risk of infection by CHIKV (Do you use the following means to protect yourself from mosquito bites?). The possible response options were 'Yes, often', 'Yes, sometimes', 'No, seldom' or 'No, never'. Of these 9 behavioural recommendations, 4 were related to biochemical control methods: using insect repellent on skin, using coils during outdoor activity, indoor insecticide spray, and indoor repellent spray. The others were related to physical interventions: sleeping under a mosquito bed net, wearing long-sleeved clothes, minimizing time outside from dusk to dawn, eliminating standing water containers, and covering water storage. To facilitate the treatment of the data concerning health protective behaviours, responses obtained from the various behavioural variables were dichotomized by combining the positive options ('Yes, often', 'Yes, sometimes') into a 'Yes' category (coded as 1), and the negative options ('No, seldom', 'No, never') into a 'No' category (coded as 0 ). For each of these 9 protective actions, the combination of these dichotomous responses enables us to distinguish between 4 trajectories characterizing participants' behaviour during the epidemic: inaction $(0,0)$, maintenance $(1,1)$, adoption $(0,1)$, and relapse $(1,0)$. Finally, the values for each item were added to generate a cumulative score (scale 0 9) that enables to measure change over time in participants' engagement in protective behaviours 
specifically recommended by the public health authorities to tackle the chikungunya epidemic (Cronbach's alpha=0.62 and 0.63 in Wave 1 and 2, respectively).

\section{Statistical Analysis}

Statistical Product and Service Solution (SPSS) 19 was used to analyse the data. Percentage and chi-square tests were calculated for all measures related to the frequency of health protective behaviours. Means and standard deviations were calculated for all measures related to risk perception. To compare differences in scores between the baseline and follow-up, $t$ tests for paired sample were performed, and effect sizes (Cohen's $d$ ) were calculated. Analyses of variance (ANOVA) were used to compare differences in score between trajectories in the participants' behavioural response to the health threat,. Pearson correlations were calculated to assess the association between changes in risk perceptions and changes in health protective behaviours,.

\section{Results}

\section{How Did Health Protective Behaviours Change During the Epidemic?}

The distribution of the behavioural trajectories related to the 9 health protective behaviours recommended by public health authorities is presented in Figure 3. First, all health protective behaviours exhibited an important degree of inertia during the epidemic, as maintenance and inaction were found to represent the behavioural response to the risk of CHIKV infection over time reported by the majority of respondents (from $71.9 \%$ to $90.7 \%$ ). However, participants were more likely to report chikungunya protective behaviours in the follow-up than in the baseline study $(M=4.17$ vs $M=3.75$; $t(433)=-5.29, p<0.001, d=.21)$, lending empirical support to the prevalence elasticity of preventive behaviours hypothesis derived from behavioural economics theory. As shown in Figure 3, participants 
were found to engage in all health protective behaviours more often or as often between the baseline and follow-up surveys, except for indoor insecticide spray use, for which the number of respondents declaring a relapse was somewhat higher than those adopting this behaviour during the course of the epidemic.

Secondly, it should be noted that a more detailed analysis of behavioural changes revealed a clear discrepancy between health protective behaviours related to individual methods of mosquito control and those related to environmental methods (see Table 3). Whereas self-reported frequency of protective behaviours associated with the environmental control method significantly increased between the baseline and the follow-up surveys $(p<0.05)$, that of personal control methods, such as using mosquito repellent, remained remarkably stable over time $(p>0.05)$.

\section{How Did the Perceived Risk Related to Chikungunya Evolve During the Epidemic?}

Participants reported a mean subjective prevalence of CHIKV infection of $35.3 \%$ in the baseline survey, and of $39.1 \%$ in the follow-up survey $(t(376)=-3.06 ; p=0.002)$, which represents a substantial deviation from the objective prevalence estimates, based on their reporting of cases within their households. Unsurprisingly, participants were indeed found to overestimate greatly the prevalence of CHIKV infection, to the extent that the number of cases that they reported in their household enables us to evaluate at about $9.5 \%$ (95\% CI: 8.5\%-10.5\%) then 14\% (95\% CI: $12.2 \%-14.6 \%)$ the objective clinical prevalence of the disease in the French Guinean population on a percentage scale [0-100]. Interestingly, as shown in Figure 2, these estimates tracked the trend in the official cumulative frequency of chikungunya cases recorded by the regional surveillance authority.

Unexpectedly, while both subjective and objective prevalence of the disease increased, participants' perceived personal risk of infection decreased from a mean of 6.8 in the baseline survey to a mean of 5.9 on the 11-point response scale in the follow-up $(t(419)=10.48 ; p<.001, d=.50)$. Overall, 
these results show the existence of a somewhat contradictory evolution between the perceived risk of infection for oneself, and that for others during the epidemic. Nevertheless, as discussed in the introduction, these paradoxical results may be attributed to either the risk reappraisal hypothesis or the risk habituation hypothesis, which could have affected the cognitive and emotional response to the health threat.

\section{Can the Risk Reappraisal Effect Account for these Paradoxical Results?}

To test empirically the risk reappraisal hypothesis, we examined the relationship between changes in protective behaviours and changes in personal risk perception. Because engaging in more health protective behaviours between the baseline and the follow-up study might have caused a reduction in the perceived risk of CHIKV infection, as well as other mosquito-borne diseases, a correlation analysis was performed between these 2 variables. Overall, the results show no evidence supporting the risk reappraisal hypothesis, as the correlation was insignificant $(r(420)=-.02, p=.676)$. Further, there was no significant correlation between changes in the perceived prevalence of CHIKV infection and those reported in health protective behaviours $(r(377)=-.00, p=.975)$.

To examine further the relationship between health behaviour changes and risk perception, we performed an analysis of variance on changes in the risk perception scale modelled by the 4 possible trajectories for each of the 9 protective behaviours recommended by the public health authorities during the epidemic (inaction, maintenance, adoption, and relapse). The results of these analyses are displayed in Table 4. People who engaged in a specific protective behaviour over time were not found to experience a decrease in their perceived risk of CHIKV infection $(p>.01)$, providing some empirical support to the risk habituation hypothesis but not to the risk appraisal hypothesis in this epidemic setting. 


\section{Discussion}

The rapid spread of the chikungunya virus across American and Caribbean countries in 201415 provided us with the opportunity to test empirically a series of hypotheses derived from the psychological and behavioural sciences regarding the complex and dynamic interactions that occur between epidemiological, behavioural and cognitive factors when a population is faced with a large outbreak of an infectious disease. Despite health psychology's growing interest in the ecological determinants of health behaviours, little attention has been given to the influence of epidemiological factors on behavioural change within modern societies, which is increasingly recognized by epidemiologists and mathematical modellers as a fundamental parameter to understand in order to predict better the course of infectious diseases (Ferguson, 2007; Funk et al., 2010). To date, the most promising hypotheses concerning the dynamic relationships between the epidemiological environment and the adoption of protective behaviour emanates from a small number of empirical studies performed by behavioural economists who have found accuracy, panic, and habituation effects as communities' responses to a health threat over time. In the field of communicable diseases, one of the best-documented phenomena is the elasticity-prevalence of protective behaviours, which can be considered as a particular manifestation of the accuracy effect (differences in health behaviours accurately reflects differences in disease prevalence). Noticeably, it has been shown that the utilisation of mosquito bed nets can be plotted as a function of the prevalence of malaria in various tropical countries (Pattanayak et al., 2007; Picone et al., 2013; Seban et al., 2013). Overall, health protective behaviours seem to be less frequent in areas or communities with lower disease prevalence. Nevertheless, as mentioned earlier, these results generally stem from aggregated data at the cross-country level, which do not enable the hypothesis of a prevalence elasticity of health behaviours to be tested directly. At the individual level, we still do not know if people really adjust their preventive actions as a function of the disease prevalence 
(Pattanayak et al., 2006). Moreover, as it has been demonstrated that people alter their protective behaviour according to how they perceive health threats, logically behavioural change should be motivated more by the perceived than the actual prevalence of disease. However, there exists to date no evidence that people from various communities or areas in which actual prevalence varied, perceived any differently the prevalence of disease in their social environment.

In addressing this gap in the literature, the current analysis of the longitudinal data we collected about risk perceptions and behaviours among French Guineans during the epidemic of Chikungunya only partially confirms, at the individual level, some of the effects that were observed at the population level. First of all, and consistent with findings from a previous study based on aggregate data conducted in tropical regions, the subjective disease prevalence reported by participants was found to track roughly the objective prevalence, as estimated by the epidemiological surveillance authority (see Figure 2). Similarly, self-reported frequency of health protective behaviours generally reflected changes in both the actual and perceived prevalence, lending empirical support to the accuracy hypothesis (see Table 3). This relationship however was not confirmed at the individual level, as behavioural changes were not found to be positively associated with the change in the perceived prevalence of CHIKV infection reported by the participants during the epidemic. This finding suggests that the elasticity prevalence effects reported in some recent studies may constitute another example of ecological fallacy. This notion refers to a common error which is likely to occur in ecological studies when "inferences about individual behaviour are drawn from data about aggregates" (Freedman, 1999). Many epidemiological studies have shown that correlations between variables at an individual level could be incorrectly deduced from correlations observed between the variables collected for the geographical area in which those individuals live (Piantadosi et al., 1988). Therefore, as proposed by (Pattanayak et al., 2006), an alternative explanation for the prevalence elasticity of protective behaviours found by behavioural economists is that this effect may instead be measuring the 
impact of the interventions implemented by public health authorities to promote a range of behaviours aimed at preventing mosquito-borne diseases, such as the distribution of bed nets in high-risk geographic areas. Interestingly, this assumption is consistent with the observed significant differences in the evolution of protective behaviours recommended by the health authorities, as those based on environmental interventions appear more responsive to the epidemiological setting than those based on individual interventions. Indeed, it should be noted that engagement in the former type of protective behaviour is substantially more visible to other people than engagement in the latter. Environmental control methods are therefore potentially more subject to a variety of social influences that are well-documented in the social and psychological sciences, such as peer pressure or stigma prevention. Indeed, many researches have shown that individuals who are perceived to pose threats to community health are likely to be identified, labelled and rejected from social interactions (Baumeister and Gitter, 2008).

Another striking finding from this longitudinal study is indisputably the downward trend observed in measures of perceived risk associated with chikungunya through the course of the epidemic (see Table 2). As an individual's heightened perception of risk is generally acknowledged to motivate engagement in health behaviours, one might have expected that both worry and perceived vulnerability would increase as the disease spread within the population. Yet, consistent with several previous studies conducted within an epidemic setting (Gidengil et al., 2012; Rubin et al., 2010; Sherlaw and Raude, 2013), risk perceptions were not found to track the disease dynamic during the outbreak, such as its prevalence or severity. Moreover, risk perceptions were not found to mirror health protective behaviours accurately over the course of the chikungunya epidemic. In recent health psychology literature, this paradoxical trend has been attributed to the risk reappraisal effect, which refers to an adaptive cognitive process whereby people are likely to readjust their perceived vulnerability to a health threat once they have taken protective actions (Brewer et al., 2007b). In the current study, however, as explained in the results section, the risk 
reappraisal hypothesis failed to account for the observed paradoxical trend of risk perceptions and health behaviours during the chikungunya outbreak in French Guiana. One possible explanation for the absence of risk reappraisal is that people living in tropical regions are more likely to maintain health protective behaviours as they are faced with numerous endemic diseases transmitted by mosquitoes, e.g. dengue fever or malaria. In other words, the participants might be exposed to a variety of cues for action that prevent significant relapse. This explanation, though, does not account for the observed differences in temporal variations in visible and invisible health protective behaviours.

Overall, our empirical results suggest that people are subject to a risk habituation effect in an epidemic setting. To date, risk habituation effects have received relatively little attention in the health psychology literature, probably because there are still relatively few longitudinal studies in health behaviour research (Sutton, 2004). Nevertheless, risk habituation effects have been the focus of more general theoretical and empirical research devoted to habituation, which refers to the psychological processes whereby repeated or prolonged exposure to a particular stimulus leads to a decreased cognitive, emotional or behavioural response, in the absence of sensory adaptation or fatigue (Thompson, 2009). Risk habituation in the current epidemic context may account for our somewhat paradoxical findings, particularly if they were to be examined in terms of Rankin et al.'s first habituation characteristics (Rankin et al., 2009). Habituation may therefore have occurred in our sample as a result of continual exposure to the stimulus of repeated health warnings in the news and social media, and/or observed cases of illness in the social circles of the participants, resulting in a decrease in cognitive and emotional response to the threat, in this case, participants' lowered perceived risk of CHIKV infection.

Furthermore, even though the risk habituation effect remains poorly studied and understood to date, its cognitive foundations may be relatively similar to those of unrealistic optimism about 
the risks of contracting an illness. As risk habituation can be viewed as a growing feeling of optimism over time, it is possible that the cognitive mechanisms underlying optimism bias also account, at least partly, for our paradoxical findings. In a series of articles, Weinstein and his colleagues (Weinstein, 1989; Weinstein et al., 2005; Weinstein and Klein, 1996) proposed four cognitive factors that may contribute to unrealistic optimism: (1) a lack of personal experience of the problem, (2) the belief that the problem may be avoided by adopting appropriate protective behaviour, (3) the belief that the problem is rare, and (4) the person's belief that if she or he had not yet been affected by the problem, she or he would remain unaffected by it. This last underlying factor of unrealistic optimism could also provide a suitable cognitive explanation for the risk habituation effect. Indeed, as most of the participants appeared to remain immune to CHIKV infection, despite the spread of the disease, they may have developed some confidence in the belief that they were unlikely to become infected during the outbreak. It should be noted here that this type of reasoning bias has long been identified and discussed by philosophers and statisticians (Jefferson, 2008; Scheutz and Poulsen, 1999; Strike, 2014). For instance, in his parable of the inductivist turkey, Bertrand Russel amusingly explains that these animals have good reason to think that humans are kind and wish them no harm, as the number of empirical observations confirming this assumption increases as Thanksgiving approaches (Russell, 2001). Applied to the results of our study, this common bias in inference and reasoning may provide a rational explanation as to why perceived personal risk paradoxically decreased over time, whereas the increase in chikungunya prevalence was accurately perceived by participants.

\section{Limitations}

This study may be subject to a variety of limitations that have been consistently observed in telephone survey, such as the discrepancy between actual and self-reported health protective 
behaviours which are attributable to the social desirability response bias. Beyond the common biases resulting from our study design, alternative or complementary explanations for the results may be found in the work conducted by behavioural economists on how individual behaviours respond to public interventions that modify exposure to arboviral diseases and their vectors (Pattanayak et al., 2006). Indeed, when government interventions are implemented to reduce the disease transmission rate in a population, people may respond by reducing their engagement in 'private' protective behaviours, resulting in a reduction in the overall effectiveness of public interventions. In their research on mosquito-borne diseases, Indira Gujral and her colleagues have observed such a tendency to reduce some key preventive behaviours on the part of the individual in cities of North Colorado where extensive government mosquito control programs had been developed (Gujral et al., 2007). According to the authors, such 'behavioural offsetting' may explain why, despite an 'actual' reduction in vector exposure in these populations, the rate of West Nile Virus infection in fact increased. This research suggests that when confronted with risk of infection, some people may progressively defer responsibility for protection to external entities. During the CHIKV epidemic, public health authorities waged a campaign which not only promoted the adoption of individual prevention measures, but also publicized government actions undertaken to preserve population health, such as 'fogging' or spraying chemicals to reduce mosquito numbers. As a result of this publicity, participants may have been likely to consider that the 'government' could effectively control the spread of the disease. This may have led people to defer responsibility for their health protection to this powerful other, thereby reducing their levels of perceived risk of infection. Thus, this externalized locus of control might partly account for the participants' limited engagement in increasing individual protective behaviour.

\section{Conclusion}


In a context of expanding emerging mosquito-borne diseases in the American tropical regions, we took advantage of the large epidemic of CHIKV infection that hit French Guiana to conduct a longitudinal study in order to develop a heightened understanding of health behaviour changes implemented by individuals in response to infectious disease outbreaks. This study highlights a series of complex interactions between human behaviours and their epidemiological environment. Firstly, it was found that people are likely to fail to adjust their risk perceptions, and to a lesser extent their health protective behaviours, to the course of an epidemic. Contrary to previous studies of the behavioural response to infectious diseases, we found that the prevalence elasticity of preventive action could not be attributed at the individual level to the perception of an increased prevalence of infection. Further, the influence of the epidemiological context on health behaviour change was found to differ substantially according to the type of measures (personal versus environmental methods). These paradoxical results can probably be attributed to risk habituation effects, which seem however to vary according to the social visibility of the protective action. This finding suggests that more attention should be given to some neglected inter-individual factors, such as stigma prevention, that may mitigate, in an epidemic setting, the influence of cognitive and emotional factors involved in risk perceptions regarding infection on the adoption or otherwise of health protective behaviours. 


\section{References}

Bajak, A., 2014. US assesses virus of the Caribbean. Nature 512, 124-125.

Baumeister, R.F., Gitter, S., 2008. I Am Approaching the Decision to Avoid You: An Approach and Avoidance Perspective on Research on Social Exclusion and Rejection. Handbook of Approach and Avoidance Motivation 601-614.

Brewer, N.T., Chapman, G.B., Gibbons, F.X., Gerrard, M., McCaul, K.D., Weinstein, N.D., 2007a. Meta-analysis of the relationship between risk perception and health behavior: the example of vaccination. Health psychology 26, 136.

Brewer, N.T., Cuite, C.L., Herrington, J.E., Weinstein, N.D., 2007b. Risk compensation and vaccination: Can getting vaccinated cause people to engage in risky behaviors? Annals of Behavioral Medicine 34, 95-99. https://doi.org/10.1007/BF02879925

Brewer, N.T., Weinstein, N.D., Cuite, C.L., Herrington, J.E., 2004. Risk perceptions and their relation to risk behavior. Annals of Behavioral Medicine 27, 125-130. https://doi.org/10.1207/s15324796abm2702_7

Burt, F.J., Rolph, M.S., Rulli, N.E., Mahalingam, S., Heise, M.T., 2012. Chikungunya: a reemerging virus. Lancet 379, 662-671. https://doi.org/10.1016/S0140-6736(11)60281-X

Cassadou, S., Boucau, S., Petit-Sinturel, M., Huc, P., Leparc-Goffart, I., Ledrans, M., 2014. Emergence of chikungunya fever on the French side of Saint Martin island, October to December 2013. Eurosurveillance 19, 6-9. https://doi.org/10.2807/15607917.ES2014.19.13.20752

Cherif, A., Barley, K., Hurtado, M., 2016. Homo-psychologicus: Reactionary behavioural aspects of epidemics. Epidemics 14, 45-53.

Dillard, A.J., Ferrer, R.A., Ubel, P.A., Fagerlin, A., 2012. Risk perception measures' associations with behavior intentions, affect, and cognition following colon cancer screening messages. Health psychology 31, 106.

Elder, J., Lloyd, L., 2006. Achieving behaviour change for dengue control: methods, scaling-up and sustainability. Report of the Scientific Working Group on Dengue 140-9.

Ferguson, N., 2007. Capturing human behaviour. Nature 446, 733.

Ferrer, R.A., Klein, W.M., 2015. Risk perceptions and health behavior. Current opinion in psychology 5, 85-89.

Ferrer, R.A., Portnoy, D.B., Klein, W.M., 2013. Worry and risk perceptions as independent and interacting predictors of health protective behaviors. Journal of health communication 18 , 397-409.

Flamand, C., Camille Fritzell, null, Pauline Obale, null, Quenel, P., Raude, J., 2017. The Role of Risk Proximity in the Beliefs and Behaviors Related to Mosquito-Borne Diseases: The Case of Chikungunya in French Guiana. Am. J. Trop. Med. Hyg. 97, 344-355. https://doi.org/10.4269/ajtmh.16-1028

Freedman, D.A., 1999. Ecological inference and the ecological fallacy. International Encyclopedia of the social \& Behavioral sciences 6, 4027-4030.

Fritzell, C., Bailly, S., Rousset, D., Kazanji, M., Raude, J., Flamand, C., 2019. Community knowledge, perceptions and behaviors related to chikungunya and Zika seropositivity (Unpublished manuscript).

Funk, S., Bansal, S., Bauch, C.T., Eames, K.T., Edmunds, W.J., Galvani, A.P., Klepac, P., 2015. Nine challenges in incorporating the dynamics of behaviour in infectious diseases models. Epidemics 10, 21-25. 
Funk, S., Salathe, M., Jansen, V.A.A., 2010. Modelling the influence of human behaviour on the spread of infectious diseases: a review. Journal of the Royal Society Interface 7, 12471256. https://doi.org/10.1098/rsif.2010.0142

Geoffard, P.-Y., Philipson, T., 1996. Rational epidemics and their public control. International economic review 603-624.

Gidengil, C.A., Parker, A.M., Zikmund-Fisher, B.J., 2012. Trends in risk perceptions and vaccination intentions: a longitudinal study of the first year of the H1N1 pandemic. American journal of public health 102, 672-679.

Golden, S.D., Earp, J.A.L., 2012. Social ecological approaches to individuals and their contexts: twenty years of health education \& behavior health promotion interventions. Health Education \& Behavior 39, 364-372.

Gujral, I.B., Zielinski-Gutierrez, E.C., LeBailly, A., Nasci, R., 2007. Behavioral risks for West Nile Virus disease, northern Colorado, 2003. Emerging Infectious Diseases 13, 419-425. https://doi.org/10.3201/eid1303.060941

Jefferson, T., 2008. More cases, doctor? Yes please! Cases Journal 1, 38. https://doi.org/10.1186/1757-1626-1-38

Lima, M.L., 2004. On the influence of risk perception on mental health: living near an incinerator. Journal of environmental psychology 24, 71-84.

Lo Presti, A., Lai, A., Cella, E., Zehender, G., Ciccozzi, M., 2014. Chikungunya virus, epidemiology, clinics and phylogenesis: A review. Asian Pacific Journal of Tropical Medicine 7, 925-932. https://doi.org/10.1016/S1995-7645(14)60164-4

Loewenstein, G., Mather, J., 1990. Dynamic processes in risk perception. Journal of Risk and Uncertainty 3, 155-175.

O’Sullivan, O., Campbell, T., Darby, A., Mourillon, N., Campbell, J., Melbourne, T., Green, V., Jackson, M.D., 2018. Chikungunya Virus Infection-associated Arthralgia in Adult Jamaicans Post-outbreak. West Indian Medical Journal 67.

Pattanayak, S., Poulos, C., Jones, K., Yang, J., Van Houtven, G., 2006. Economics of Environmental Epidemiology. RTI Working Paper 06_04. Research Triangle Park, NC: Research Triangle Institute.

Pattanayak, S.K., Poulos, C., Van Houtven, G., Yang, J.-C., Jones Wendland, K., 2007. Modeling Self-Protective Behaviors Against Infectious Disease: Estimates of Prevalence Elasticity for Malaria.

Philipson, T., 2000. Economic epidemiology and infectious diseases. Handbook of health economics 1, 1761-1799.

Pialoux, G., Gaüzère, B.-A., Jauréguiberry, S., Strobel, M., 2007. Chikungunya, an epidemic arbovirosis. The Lancet Infectious Diseases 7, 319-327. https://doi.org/10.1016/S14733099(07)70107-X

Piantadosi, S., Byar, D.P., Green, S.B., 1988. The ecological fallacy. American journal of epidemiology 127, 893-904.

Picone, G., Kibler, R., Apouey, B.H., 2013. Malaria prevalence, indoor residual spraying, and insecticide-treated net usage in Sub-Saharan Africa.

Rankin, C.H., Abrams, T., Barry, R.J., Bhatnagar, S., Clayton, D.F., Colombo, J., Coppola, G., Geyer, M.A., Glanzman, D.L., Marsland, S., 2009. Habituation revisited: an updated and revised description of the behavioral characteristics of habituation. Neurobiology of learning and memory 92, 135-138. 
Raude, J., Peretti-Watel, P., Ward, J., Flamand, C., Verger, P., 2018. Are Perceived Prevalences of Infection also Biased and How? Lessons from Large Epidemics of Mosquito-Borne Diseases in Tropical Regions. Med Decis Making 38, 377-389. https://doi.org/10.1177/0272989X17750845

Robinson, M.C., 1955. An epidemic of virus disease in Southern Province, Tanganyika Territory, in 1952-53. I. Clinical features. Trans. R. Soc. Trop. Med. Hyg. 49, $28-32$.

Rogers, G.O., 1997. The dynamics of risk perception: How does perceived risk respond to risk events? Risk Analysis 17, 745-757.

Rougeron, V., Sam, I.-C., Caron, M., Nkoghe, D., Leroy, E., Roques, P., 2015. Chikungunya, a paradigm of neglected tropical disease that emerged to be a new health global risk. Journal of clinical Virology 64, 144-152.

Rubin, G.J., Potts, H.W.W., Michie, S., 2010. The impact of communications about swine flu (influenza A H1N1v) on public responses to the outbreak: results from 36 national telephone surveys in the UK. Health Technology Assessment 14, 183-266.

Russell, B., 2001. The problems of philosophy. OUP Oxford.

Sallis, J.F., Owen, N., Fisher, E., 2015. Ecological models of health behavior. Health behavior: Theory, research, and practice 5, 43-64.

Scheutz, F., Poulsen, S., 1999. Determining causation in epidemiology. Community dentistry and oral epidemiology $27,161-170$.

Seban, J., Thuilliez, J., Herbreteau, V., 2013. Possession of bed nets in Haut-Katanga (DRC): Prevalence-elastic behaviour or performance of health care system delivery? Health \& place 24, 275-285.

Sheeran, P., Harris, P.R., Epton, T., 2014. Does heightening risk appraisals change people's intentions and behavior? A meta-analysis of experimental studies. Psychological bulletin 140, 511.

Sherlaw, W., Raude, J., 2013. Why the French did not choose to panic: a dynamic analysis of the public response to the influenza pandemic. Sociology of health \& illness 35, 332-344.

Sidi, Y., Harel, O., 2018. The treatment of incomplete data: Reporting, analysis, reproducibility, and replicability. Social Science \& Medicine 209, 169-173. https://doi.org/10.1016/j.socscimed.2018.05.037

Simon, F., Javelle, E., Oliver, M., Leparc-Goffart, I., Marimoutou, C., 2011. Chikungunya virus infection. Current infectious disease reports 13, 218.

Strike, P.W., 2014. Statistical methods in laboratory medicine. Butterworth-Heinemann.

Sutton, S., 2004. Determinants of Health-Related Behaviours: Theoretical \& Methodological Issues., in: Sutton, Stephen, Baum, A., Johnston, M. (Eds.), The SAGE Handbook of Health Psychology. SAGE, London, pp. 94-126.

Thompson, R.F., 2009. Habituation: a history. Neurobiology of learning and memory 92, 127.

Verelst, F., Willem, L., Beutels, P., 2016. Behavioural change models for infectious disease transmission: a systematic review (2010-2015). Journal of The Royal Society Interface 13, 20160820.

Weaver, S.C., 2014. Arrival of Chikungunya Virus in the New World: Prospects for Spread and Impact on Public Health. PLoS Negl Trop Dis 8. https://doi.org/10.1371/journal.pntd.0002921

Weinstein, N.D., 2000. Perceived probability, perceived severity, and health-protective behavior. Health Psychology 19, 65.

Weinstein, N.D., 1989. Optimistic biases about personal risks. Science 246, 1232-1234. 
Weinstein, N.D., Klein, W.M., 1996. Unrealistic optimism: Present and future. Journal of Social and Clinical Psychology 15, 1-8.

Weinstein, N.D., Kwitel, A., McCaul, K.D., Magnan, R.E., Gerrard, M., Gibbons, F.X., 2007. Risk perceptions: assessment and relationship to influenza vaccination. Health Psychology 26, 146.

Weinstein, N.D., Marcus, S.E., Moser, R.P., 2005. Smokers' unrealistic optimism about their risk. Tobacco control 14, 55-59.

Weinstein, N.D., Nicolich, M., 1993. Correct and incorrect interpretations of correlations between risk perceptions and risk behaviors. Health psychology 12, 235.

Weston, D., Hauck, K., Amlôt, R., 2018. Infection prevention behaviour and infectious disease modelling: a review of the literature and recommendations for the future. BMC public health $18,336$.

WHO, 2004. Global strategic framework for integrated vector management [WWW Document]. WHO. https://doi.org//entity/malaria/publications/atoz/who_cds_cpe_pvc_2004_10/en/index.ht $\mathrm{ml}$

Xu, J., Peng, Z., 2015. People at Risk of Influenza Pandemics: The Evolution of Perception and Behavior. PLOS ONE 10, e0144868. https://doi.org/10.1371/journal.pone.0144868 
Figure 1. Models of dynamical relations between disease prevalence and risk perception adapted from the work by Loewenstein and Mather (1990)

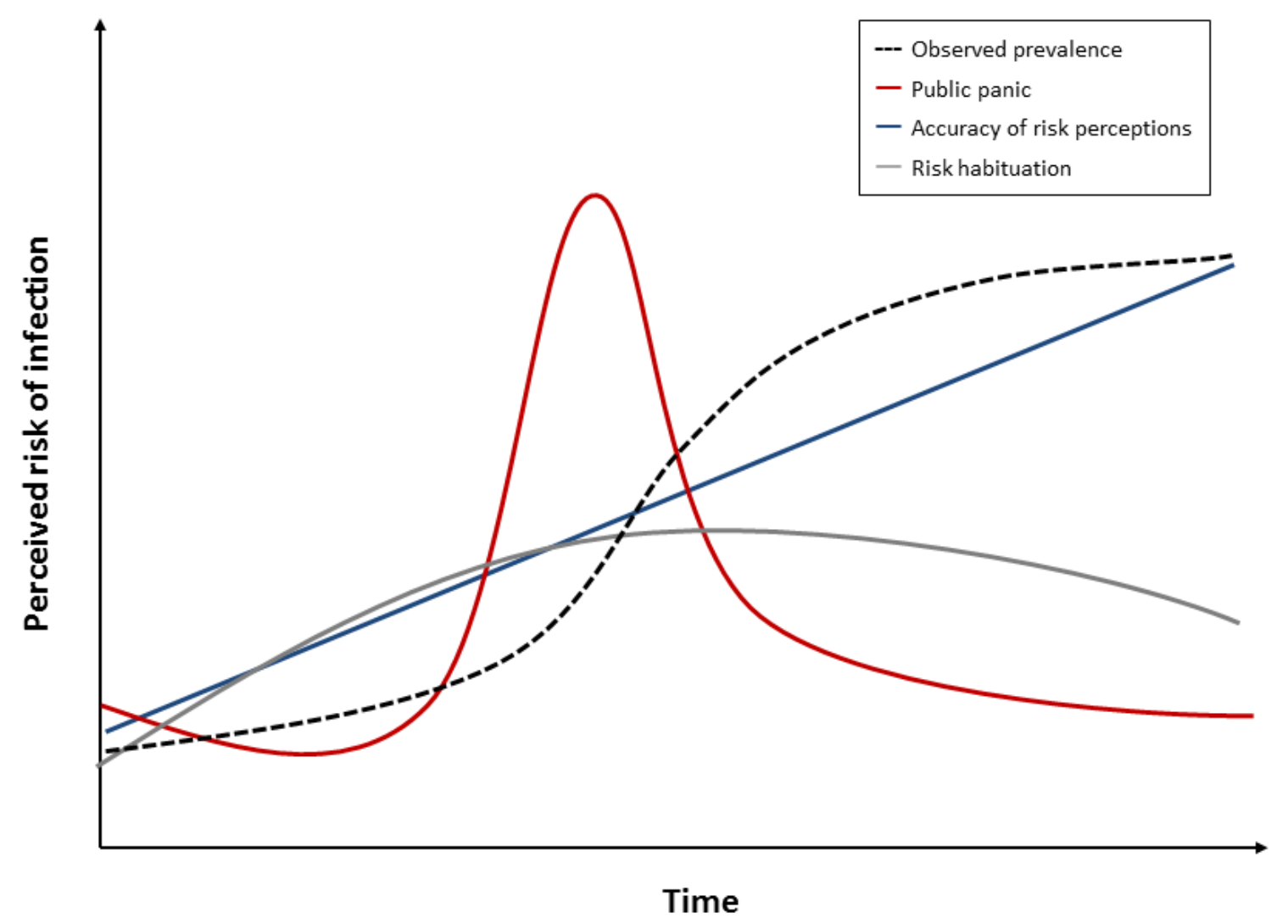


Figure 2. The cumulative frequency of chikungunya cases in French Guiana based on the observational data collected by the regional system of epidemiological surveillance.

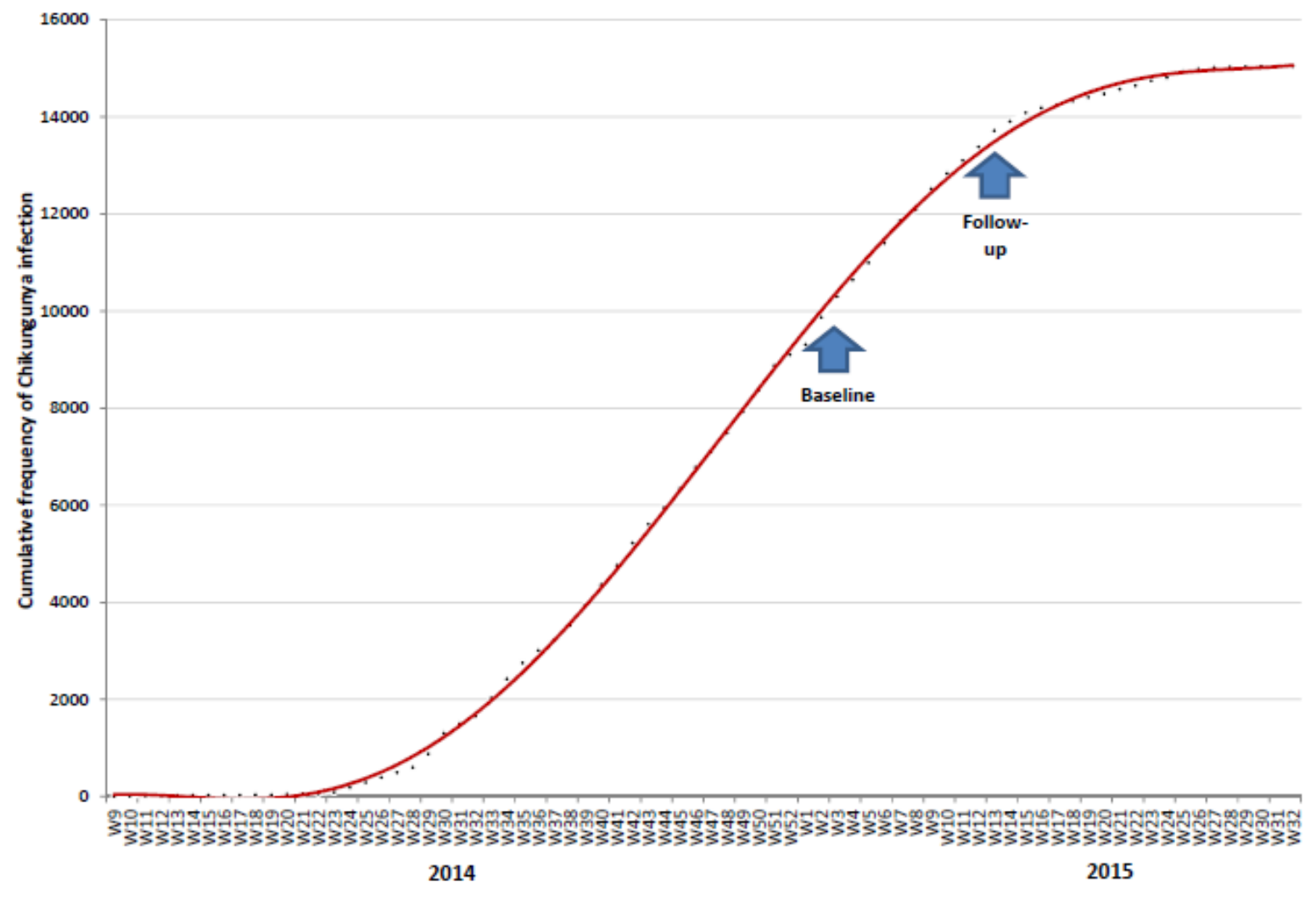


Figure 3. Trajectories over time of the 9 health protective behaviours recommended by the public health authorities.

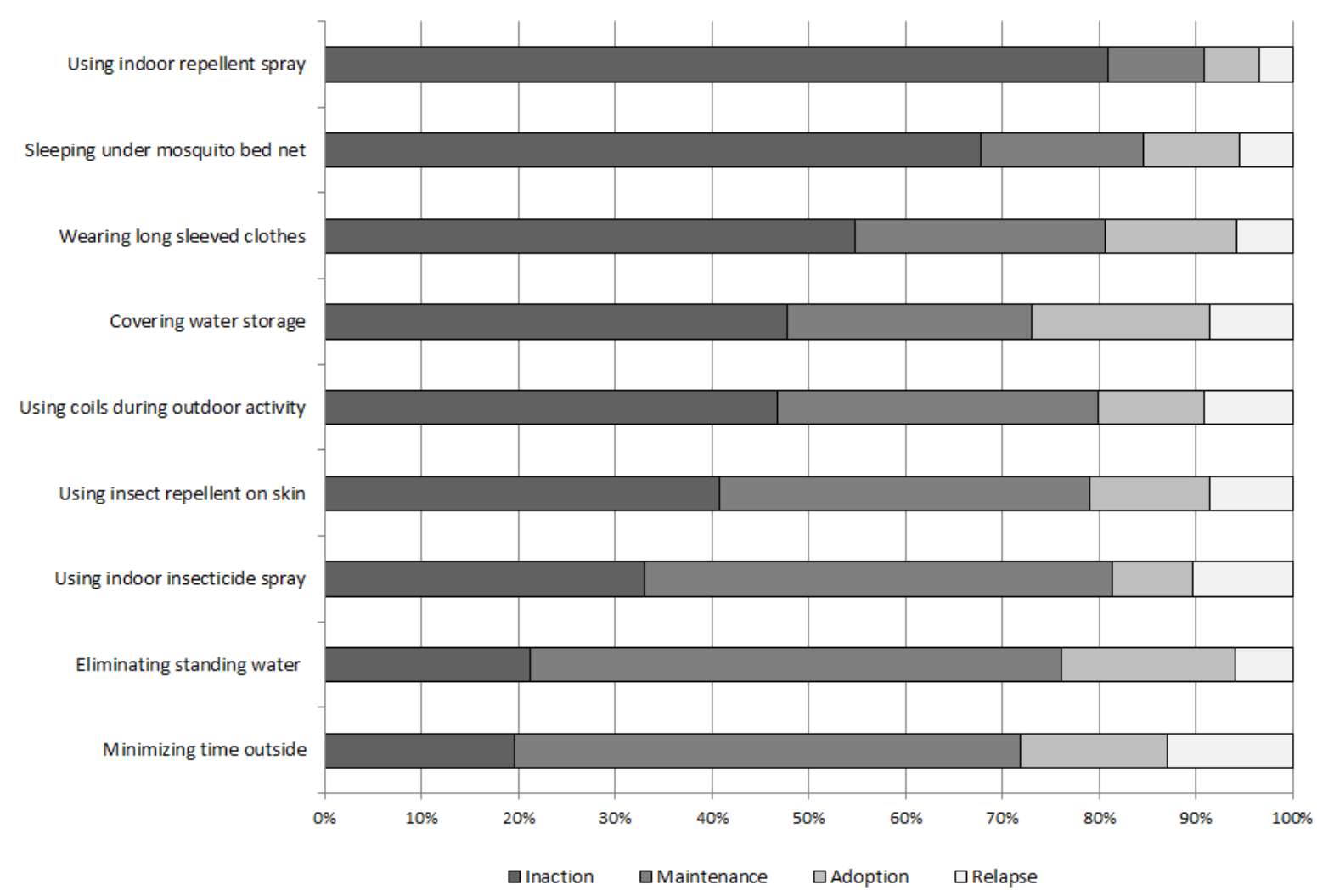


Table 1. Sociodemographic characteristics of the samples (percentage and frequencies)

\begin{tabular}{|c|c|c|c|c|}
\hline Characteristics & $\begin{array}{l}\text { Baseline } \\
(\mathrm{N}=768)\end{array}$ & $\begin{array}{l}\text { Follow-up } \\
(\mathrm{N}=434)\end{array}$ & $c^{2}(d f, N)$ & $p$-value \\
\hline Geographic Area & & & $1.65(3,1,202)$ & 0.648 \\
\hline Cayenne & $30.2 \%(232)$ & $30.6 \%(133)$ & & \\
\hline Kourou-Macouria & $17.4 \%(134)$ & $15.4 \%(67)$ & & \\
\hline Mana-Saint Laurent-Sinamary & $24.1 \%(185)$ & $22.8 \%(99)$ & & \\
\hline Other geographic areas & $28.3 \%(217)$ & $31.1 \%(135)$ & & \\
\hline Sex & & & $1.11(1,1,202)$ & 0.292 \\
\hline Male & $47.5 \%(365)$ & $50.7 \%(220)$ & & \\
\hline Female & $52.5 \%(403)$ & $49.3 \%(214)$ & & \\
\hline Age & & & $3.79(4,1,202)$ & 0.433 \\
\hline 18-24 years old & $17.6 \%(135)$ & $18.0 \%(78)$ & & \\
\hline 25-34 years old & $23.6 \%(181)$ & $18.9 \%(82)$ & & \\
\hline $35-44$ years old & $22.8 \%(175)$ & $24.7 \%(107)$ & & \\
\hline $45-59$ years old & $24.6 \%(189)$ & $25.6 \%(111)$ & & \\
\hline$\geq 60$ & $11.5 \%(88)$ & $12.9 \%(56)$ & & \\
\hline Employment status & & & $.83(4,1,202)$ & 0.934 \\
\hline Employed or self-employed & $49.7 \%(382)$ & $49.3 \%(214)$ & & \\
\hline Unemployed & $16.7 \%(128)$ & $16.4 \%(71)$ & & \\
\hline Student & $12.0 \%(92)$ & $12.0 \%(52)$ & & \\
\hline Retired & $8.2 \%(63)$ & $9.7 \%(42)$ & & \\
\hline Other status & $13.4 \%(103)$ & $12.7 \%(55)$ & & \\
\hline Size of household & & & $1.63(4,1202)$ & 0.803 \\
\hline 1 person & $73(9.5 \%)$ & $34(7.8 \%)$ & & \\
\hline 2 persons & $137(17.8 \%)$ & $78(18.0 \%)$ & & \\
\hline 3 persons & $153(19.9 \%)$ & $86(19.8 \%)$ & & \\
\hline 4 persons & $181(23.6 \%)$ & $113(26.0 \%)$ & & \\
\hline$\geq 5$ persons & $224(29.2 \%)$ & $123(28.3 \%)$ & & \\
\hline Type of housing & & & $.30(3,1202)$ & 0.960 \\
\hline Individual modern house & $63.9 \%(491)$ & $65.2 \%(283)$ & & \\
\hline Collective modern house & $29.4 \%(226)$ & $28.8 \%(125)$ & & \\
\hline Individual traditional house & $4.4 \%(34)$ & $3.9 \%(17)$ & & \\
\hline Other type of housing & $2.2 \%(17)$ & $2.1 \%(9)$ & & \\
\hline Type of community & & & $.26(3,1202)$ & 0.967 \\
\hline
\end{tabular}




\begin{tabular}{llll} 
City or urban community & $55.2 \%(424)$ & $56.7 \%(246)$ & \\
Suburban community & $21.7 \%(167)$ & $21.2 \%(92)$ & \\
Rural community & $22.5 \%(173)$ & $21.7 \%(94)$ & \\
Other type of community & $0.5 \%(4)$ & $0.5 \%(2)$ & $.05(1,202)$ \\
Personal history of chikungunya & & & \\
Yes & $10.5 \%(80)$ & $10.8 \%(47)$ & \\
No/Unsure & $89.5 \%(688)$ & $89.2 \%(387)$ & \\
\hline
\end{tabular}

${ }^{*} \mathrm{p}<0.05,{ }^{* *} \mathrm{p}<0.01,{ }^{* * *} \mathrm{p}<0.001$ 
Table 2. Perceptions of risk related to chikungunya: means, standard deviations, and paired sample tests.

\begin{tabular}{llccc}
\hline Variables & Baseline & Follow-up & $\boldsymbol{t}$-value $(\boldsymbol{d}$ f) & $\boldsymbol{p}$-value \\
\hline Perceived prevalence (0-100) & $35.9(25.6)$ & $39.2(25.7)$ & $-3.06(376)$ & $.002^{* *}$ \\
Chikungunya-related worry (0-10) & $7.16(3.02)$ & $6.24(3.13)$ & $5.87(430)$ & $<.001^{* * *}$ \\
Perceived exposure to mosquitos (0-10) & $6.40(2.90)$ & $6.29(2.82)$ & $.69(433)$ & .490 \\
Perceived Vulnerability (0-10) & $6.48(2.78)$ & $5.62(2.85)$ & $5.38(427)$ & $<.001^{* * *}$ \\
Perceived risk of infection (0-10) & $6.59(2.60)$ & $3.90(2.42)$ & $17.54(433)$ & $<.001^{* * *}$ \\
Perceived Severity (0-10) & $7.50(2.08)$ & $7.56(1.93)$ & $-.48(429)$ & .633 \\
\hline Risk perception scale (0-10) & $6.84(1.77)$ & $5.90(1.82)$ & $10.48(419)$ & $<.001^{* * *}$ \\
\hline
\end{tabular}

${ }^{*} \mathrm{p}<0.05,{ }^{* *} \mathrm{p}<0.01,{ }^{* * *} \mathrm{p}<0.001$ 
Table 3. Self-reported frequency of health protective behaviours: percentages (frequencies), Pearson chi-square, and $p$-value.

\begin{tabular}{|c|c|c|c|c|}
\hline Behaviour & Baseline $(N=768)$ & Follow-Up $(N=434)$ & $c^{2}(d f, N)$ & $p$-value \\
\hline \multicolumn{5}{|c|}{ Chemical control methods } \\
\hline Repellents & & & $.85(1,1202)$ & .355 \\
\hline Often/Sometime & $47.9 \%(368)$ & $50.7 \%(220)$ & & \\
\hline Rarely/Never & $52.1 \%(400)$ & $49.3 \%(214)$ & & \\
\hline Punctual indoor spraying & & & $.00(1,1202)$ & .989 \\
\hline Often/Sometime & $56.8 \%(436)$ & $56.7 \%(246)$ & & \\
\hline Rarely/Never & $43.2 \%(332)$ & $43.3 \%(188)$ & & \\
\hline Residual indoor spraying & & & $.03(1,1202)$ & .874 \\
\hline Often/Sometime & $16.1 \%(124)$ & $15.7 \%(68)$ & & \\
\hline Rarely/Never & $83.9 \%(644)$ & $84.3 \%(366)$ & & \\
\hline Punctual outdoor spraying & & & $2.96(1,1202)$ & .085 \\
\hline Often/Sometime & $39.1 \%(300)$ & $44.0 \%(191)$ & & \\
\hline Rarely/Never & $60.9 \%(468)$ & $56.0 \%(243)$ & & \\
\hline
\end{tabular}

Physical control methods

\begin{tabular}{lcccc}
\hline Bed nets & & $2.68(1,1202)$ & .102 \\
Often/Sometime & $22.5 \%(173)$ & $26.7 \%(116)$ & & \\
$\begin{array}{l}\text { Rarely/Never } \\
\text { Draining stagnant water }\end{array}$ & $77.5 \%(595)$ & $73.3 \%(318)$ & & \\
Often/Sometime & $60.2 \%(462)$ & $72.8 \%(316)$ & & \\
Rarely/Never & $39.8 \%(306)$ & $27.2 \%(118)$ & & \\
Covering tanks & & & $13.64(1,1202)$ & $<0.001^{* * * *}$ \\
Often/Sometime & $33.1 \%(254)$ & $43.8 \%(190)$ & & \\
Rarely/Never & $66.9 \%(514)$ & $56.2 \%(244)$ & & \\
Minimizing time outside & & & & \\
Often/Sometime & $65.0 \%(499)$ & $67.5 \%(293)$ & & \\
Rarely/Never & $35.0 \%(269)$ & $32.5 \%(141)$ & & \\
Wearing long sleeved clothes & & & \\
Often/Sometime & $32.6 \%(250)$ & $39.4 \%(171)$ & & \\
Rarely/Never & $67.4 \%(518)$ & $60.6 \%(263)$ & & \\
\hline
\end{tabular}

${ }^{*} \mathrm{p}<0.05,{ }^{* *} \mathrm{p}<0.01,{ }^{* * *} \mathrm{p}<0.001$ 
Table 4. Changes in risk perceptions modelled by behavioural trajectories during the epidemic for each of the 9 protective behaviours recommended by the public health authorities: Means (standard deviations), $F$-value (degrees of freedom) and $p$-value

\begin{tabular}{llllllr}
\hline Health protective behaviour & Inaction & Maintenance & Adoption & Relapse & $\boldsymbol{F}$ (df) & $\boldsymbol{p}$-value \\
\hline Using insect repellent on skin & $-1.17(1.82)$ & $-.89(1.77)$ & $-1.00(1.99)$ & $-.51(2.07)$ & $1.59(3,424)$ & .190 \\
Using coils during outdoor activity & $-1.05(1.96)$ & $-.89(1.59)$ & $-.90(1.97)$ & $-1.07(1.98)$ & $.24(3,424)$ & .868 \\
Using indoor insecticide spray & $-.96(1.82)$ & $-1.05(1.76)$ & $-1.23(2.30)$ & $-.51(1.92)$ & $1.27(3,424)$ & .285 \\
Using indoor repellent spray & $-1.01(1.86)$ & $-.69(1.57)$ & $-.76(1.92)$ & $-1.48(2.23)$ & $.85(3,424)$ & .466 \\
Sleeping under mosquito bed net & $-1.02(1.89)$ & $-.99(1.82)$ & $-.87(1.89)$ & $-.69(1.46)$ & $0.28(3,424)$ & .840 \\
Wearing long sleeved clothes & $-1.08(1.93)$ & $-.86(1.73)$ & $-1.16(1.66)$ & $-.22(1.92)$ & $1.98(3,424)$ & .116 \\
Minimizing time outside & $-1.30(1.48)$ & $-.79(1.80)$ & $-.90(2.11)$ & $-1.34(2.12)$ & $2.36(3,424)$ & .071 \\
Eliminating standing water & $-1.39(1.69)$ & $-.86(1.77)$ & $-.82(2.08)$ & $-1.10(2.25)$ & $2.03(3,424)$ & .109 \\
Covering water storage & $-1.06(1.85)$ & $-1.17(1.80)$ & $-.80(1.89)$ & $-.40(1.83)$ & $1.90(3,424)$ & .129 \\
\hline
\end{tabular}

